\title{
Outlines for a Phenomenological Foundation For de Ronde's Theory of Powers and Potentia
}

\author{
Matías Graffigna \\ Graduiertenschule für Geisteswissenschaften Göttingen \\ Georg-August-Universität Göttingen - Germany.
}

\begin{abstract}
Starting with the claim that Quantum Mechanics (QM) is in need of a new interpretation that would allow us to understand the phenomena of this realm, I wish to analyse in this paper de Ronde's theory of power and potentia from a phenomenological perspective. De Ronde's claim is that the reason for the lack of success in the foundations of QM is due to the reluctance of both physicists and philosophers to explore the possibility of finding a new ontology, new concepts for the physical theory. De Ronde proposes such new ontology and the question I wish to address here, is whether his ontology is conceptually plausible. I will, for this purpose, recur to Edmund Husserl's phenomenology. After presenting some of the basic concepts and methodological tools of this theory, I shall apply them to de Ronde's ontology to determine the viability of his theory.
\end{abstract}

Keywords: quantum mechanics, phenomenology, immanent powers, potentia.

\section{Introduction}

In this article, I wish to analyze from a philosophical perspective - the phenomenological in particular - one possible approach to the foundations of Quantum Mechanics (henceforth QM). Taking as a starting point the premise that QM is in need of an interpretation that would allow us not only to carry out successful experiments, but also that will grant us the possibility of understanding comprehensively the phenomena the theory describes and explains, ${ }^{1}$ it is that I wish to examine one particular approach to this problem: de Ronde's theory of powers and potentia. De Ronde's claim, to anticipate, is essentially that most of the theoretical problems QM faces nowadays are due to misunderstandings at the basic conceptual level, and the reluctance of physicists, since the early days of the theory, to adopt new metaphysical/ontological principles that suit the new theory, rather than keep insisting in trying to adapt QM to the 'classical picture' of the world that stems from Aristotelian metaphysics and Newtonian classical mechanics.

\footnotetext{
${ }^{1}$ For this discussion, see $[3$, pp. 54-55], [24, p. 230], [17, p. 2], [6, p. 9]; and my own [18, pp. 3-5]
} 
In order to do this, I will divide this work in four parts: first, I will discuss the situation concerning the relation between ontology/metaphysics and physics, to elucidate the notion of an alternative ontology, and will offer some examples of proposals that go in this line; I will then introduce de Ronde's alternative ontology for QM, mainly his concepts of power and potentia and how they stand in reference to the classical metaphysical concepts; in the third section I shall offer a very schematic and introductory presentation of some basic phenomenological concepts; and, finally, I shall try to apply these phenomenological concepts to de Ronde's ontology. The thesis I will try to defend is humble in its reach: it is my claim that de Ronde's ontology is conceptually plausible from a phenomenological perspective. Whether or not it is the right ontology for QM is something that exceeds my present purpose. Yet, I believe the claim is not trivial, inasmuch de Ronde's proposal for an alternative ontology is highly controversial, due to the fact that it contradicts several basic intuitions regarding our understanding of physical reality.

\section{What is an alternative ontology?}

De Ronde's theory is presented as an 'alternative ontology'. We could begin by asking ourselves, alternative to what? The first answer to this question would be one of a very general nature: to classical ontology. Classical ontology, or even classical metaphysics, is not a theory presented and defended by one author, but rather a series of principles, beliefs and suppositions that have settled through time in western thought, philosophy and science. There are multiple ways to understand this history and multiple ways to reconstruct what a classical ontology is. Since the discussion we are facing is one concerning the philosophy of physics, of QM in particular, it is not of the utmost importance to unequivocally determine what these principles are and where do they stem from. What is important is to understand and determine what it is that physics has taken to be classical ontology.

If we then take classical ontology to be the set of basic metaphysical principles that determine a basic worldview, upon which classical mechanics will be based in order to be developed as the standard theory that we know today, it would seem we need to go back to Aristotle. But let us begin from the 'end': classical Newtonian mechanics is based upon a series of metaphysical presuppositions that are well known by both philosophers and physicists: time and space are absolute; that which is real, which the theory describes, explains and predicts the properties and states of bodies that inhabit this space-time, and whose objective properties can be given at any time (mass, acceleration, speed, position, etc.). In other words: given a body, a real being, in classical Euclidean space, and given some initial conditions, Newtonian mechanics, is able to calculate and predict the objective properties of said body. This is nowadays so obvious that even a school child can grasp it. Now, in what sense do we claim that these basic principles of physics are based upon a classical ontology?

"One of the first such metaphysical systems, which still today plays a major role in our understanding of the world around us is that proposed by Aristotle through his logical and ontological principles: the Principle of Existence (PE), the Principle of Non-Contradiction (PNC)and the Principle of Identity (PI)" [8, p. 2]. These principles, as formulated by Aristotle, constitute the building blocks of classical metaphysical thinking, and hence, the basis upon which classical mechanics is grounded. What they do, is basically determine the notion of entity, which we can understand as: a real existent in space-time, with definite non-contradictory properties that can be known, that has an identity with itself, in the sense that it is the same entity throughout time. But these principles do not only determine an entity in a positive sense, they also exclude from the ontology everything else: something is either an entity, a property of an entity, or it doesn't belong to the realm of the physical, i.e., real existence, susceptible of physical scientific study. It would, of course, be an 
overstatement to say that all western metaphysical thinking can be reduced to these Aristotelian principles; that is not the point. The claim is that these are the ontological foundations upon which Newton developed his physical theory and since then, they have become the basic ontological presuppositions for physicists: "It was Isaac Newton who was able to translate into a closed mathematical formalism both, the ontological presuppositions present in Aristotelian (Eleatic) logic and the materialistic ideal of res extensa, with actuality as its mode of existence." [Op. cit., p. 4]

It was Kant who would later offer what he understood as the transcendental justification of the possibility of scientific knowledge [23]. Put very simply, an object of possible experience is that from which we receive sensible impressions under the pure forms of sensibility (space-time) and that falls under the transcendental categories of understanding. Everything that does not meet these two conditions is not an object of possible experience and, hence, is not an object of science, but of speculative metaphysics. We can see how with his philosophy, what we are presenting as classical ontology obtains a transcendental foundation.

Some years later, Logical Positivism, as the heir of British empiricism and German logicism, would determine the basic principles upon which our contemporary notion of science is based: what is real and scientifically knowable are entities, objects of possible experience and these are observable, inasmuch as there is a definite process by which they can be empirically determined. This is what is known as the operationalist definition of concepts, or methodology: no concept can claim scientific validity, unless there is a definite process that allows to empirically measure the concept in a series of finite steps. "The notion of operational definition thus lies at the heart of contemporary physics" [5, p. 26] and thus limits the realm of the physically real to a materialist-empiricist ontology. Everything else, once again, is outside the realm of the real, of the scientific and, thus, is nothing more than metaphysical speculation, only this time, "metaphysical" is synonym of an illegitimate form of knowledge.

Now, beyond the criticisms and discussions that could arise from any of these theories in particular, the problem that we face at present is the one brought about by the appearance of QM in the early 20th century. It is the basic principles of this theory, in its mathematical formalism and its unprecedented empirical success, that challenge our very understanding of what it means for something to be real, in particular, to be physically real.

Heisenberg's principle of indetermination, superposition states, entanglement, contextuality and, in general, all the phenomena belonging to the domain of QM, seem to contradict this classical picture of the world which was so obvious and unquestionable until not so long ago. The peculiar situation with QM is that the theory was developed first in its mathematical formalism, it was successfully taken to the labs, but until today lacks a conceptual framework: One that would allows us to understand the domain described by the theory beyond its abstract mathematical formalism and more comprehensively than in its isolated experimental results - which, as it's always the case with experimental data, need a conceptual interpretation and understanding. In the words of Griffiths:

Scientific advances can significantly change our view of what the world is like, and one of the tasks of the philosophy of science is to take successful theories and tease out of them their broader implications for the nature of reality. Quantum mechanics, one of the most significant advances of twentieth century physics, is an obvious candidate for this task, but up till now efforts to understand its broader implications have been less successful than might have been hoped. The interpretation of quantum theory found in textbooks, which comes as close as anything to defining "standard" quantum mechanics, is widely regarded as quite unsatisfactory. Among philosophers of science this opinion is almost universal, and among practicing physicists it is widespread. It is but a slight 
exaggeration to say that the only physicists who are content with quantum theory as found in current textbooks are those who have never given the matter much thought, or at least have never had to teach the introductory course to questioning students who have not yet learned to "shut up and calculate!" [17, p. 2]

The lack of a commonly accepted interpretation of the Quantum Theory is also remarked by Dorato, who also strives to find an alternative understanding of the ontological principles for QM:

One of the most frequent points of misunderstanding between physicists and philosophers of physics or metaphysicians is not only caused by differences in language but also by the fact that philosophers worry much more than physicists about ontological issues, namely interpretive questions involving what (typically a poorly understood) physical theory tells us about the world. In the case of quantum mechanics, however, interpretive questions calling for ontological analyses ("how could the world be like if quantum theory is true?") become murky since, at least according to philosophers, it is still controversial how quantum theory should be formulated, given that in the market there are various proposals. [13, p. 370]

Now, where does the cause for this failure lie? How is it that after one century physics has been unable to find meaning for these discoveries? As always, there are multiple possible answers to this question. One of them is, in the line of instrumentalists such as Fuchs \& Peres (Cf. [16]), to renounce to the question altogether and understand QM as a mere tool to produce calculations and predictions. I believe the best answer to this question, following de Ronde's line of research (see specially $[6,8,9,10])$, is to say that all attempts to offer an interpretation of QM have the same common ground: they all try to do so limiting themselves to a classical ontology. Ever since Bohr claimed that "the unambiguous interpretation of any measurement must be essentially framed in terms of classical physical theories, and we may say that in this sense the language of Newton and Maxwell will remain the language of physicists for all time" [27, p. 7], it has become a dogma for physicists and philosophers to constraint any attempt to bring meaning to QM to the very closed limits of classical metaphysical principles. This is so up to the point that physicists have decided to modify the mathematical formalism of the theory, like in Bohm's case, rather than trying to search for a different ontology. Moreover, the triumph of the scientific program of logical positivism has settled that ontology as the only possible one and precluded the possibility of having metaphysical discussions, naively believing that materialistic experimental science is free of metaphysics, just because they chose a specific set of ontological principles instead of some other. So, the situation is as tricky as it can be historically appreciated: there's only the classical ontology to serve as basis for physical theories (Bohrian lines of inquiry); discussing ontology/metaphysics is an unscientific enterprise and should be avoided (logical positivism). The result: a century of failed attempts to comprehend QM.

It seems, then, that both these constraints need to be overcome in order to have a chance to move forwards. And hence, the idea of an alternative ontology for QM. Now, of course, there is no guarantee that this path shall bring us success at the end, but there seems to be already enough guarantee that the other one has failed. Developing an alternative ontology has problems of its own, some could even be considered more challenging than the ones faced up to now, and this shall be the focus of the rest of this article.

I wish to conclude this section by mentioning three examples of proposals that go in this direction. Unfortunately, I will not be discussing them here, but they shall serve nonetheless to illustrate the general project of developing an alternative understanding for the ontological foundations of QM. In the first place, the dispositionalist account of powers in QM is worth mentioning. French 
offers such an account and acknowledges as well the need for a metaphysical discussion regarding physical theories. Yet, there is a crucial difference between his approach and my understanding of de Ronde's, as we shall see, which lies in the role itself that metaphysics are called to play: "I have tried to articulate what we have called the 'toolbox' approach to metaphysics, according to which metaphysics can be viewed as providing a set of tools that philosophers in other sub-disciplines, and particularly, the philosophy of science, can use for their own purposes" [15, p. 1]. The concept of disposition is one of such 'tools' French sets out to implement. Such a conception is, in my opinion, not free of some serious risks. We could very well call this position an instrumentalist approach to ontology, inasmuch as ontology is not the basis upon which scientific concepts are developed, but the source of ad hoc utilizations of different metaphysical concepts.

In a closer line to our own, Esfeld actually proposes a different ontological grounding for QM, with a realist aim:

Ontic structural realism is a current in contemporary metaphysics of science that maintains that in the domain of fundamental physics, there are structures in the first place rather than objects with an intrinsic identity. Its main motivation is to develop a tenable version of scientific realism in form of an ontology that meets the challenges of modern physics, giving an account of entanglement in quantum physics and of space-time in the theory of general relativity. The claim is that there are structures of entanglement instead of objects with an intrinsic identity in the domain of quantum physics (Ladyman 1998, French \& Ladyman 2003, Esfeld 2004) and metrical structures, which include the gravitational energy, instead of space-time points with an intrinsic identity in the domain of the theory of general relativity. [14, p. 1]

The last attempt I wish to mention is Dorato's proposal for an event ontology, that would replace the ontology of objects used in order to interpret the quantum formalism. This ontology also seeks to be a foundation for a realist physical science, the conclusions of which are of high interest. After presenting the different alternatives for an event ontology and their connection to QM, Dorato offers, among others, the following conclusion: "The point I want to urge is that it is possible to claim that the individual particle has no definite spin in a given direction, but that it manifests an intrinsic disposition or has a concrete power to display spin up or spin down by interacting with the Stern-Gerlach apparatus (the stimulus of the disposition)" [13, p. 376]. These two quotes exemplify what it means to search for an alternative ontology: a fundamentally different metaphysical basis for physics should have as a result a fundamentally different understanding of the quantum phenomena. It concerns the question of what it is that we describe and explain through QM, and has the explicit aim of achieving a deeper understanding that could have actual consequences for the way in which physics is done today.

\section{De Ronde's theory of immanent powers and potentia}

In this section I will present de Ronde's alternative ontology for the interpretation of QM, which is, as must be warned, a work in progress. The purpose of the present work is not to determine whether or not this ontology is, as a matter of fact, the "correct" ontology for QM, but rather to analyze its plausibility from a purely philosophical perspective. In this sense, it might well be the case that even if this proposal turned out to be philosophically solid and acceptable, it could still not be the appropriate interpretation for QM. This last item, of no minor importance, will not be

considered here. Yet, I consider the philosophical plausibility of the theory to be logically prior to its adequacy, and hence the relevance of the present work. 
De Ronde presents the basic tenants of his theory in direct contraposition to Aristotle's basic metaphysical principles that serve as the underlying base for classical mechanics:

The first important point according to our stance is to recall the fact that Aristotle grounded the notion of entity in the logical and ontological principles of existence, noncontradiction and identity. Our proposal is that in fact there exist analogous principles in QM which can allow us to develop new concepts. The principles of indetermination, superposition and difference could be considered as providing the logical and ontological foundation of that of which QM is talking about. [7, p. 11].

As Aristotle's metaphysical principles determined the concept of entity, which is central both to his philosophy and to the following history of western thought, de Ronde proposes three new different principles that basically stem from the quantum theory itself. We shall focus on each one of them later, the point to be made now is that from these principles, that are 'no more' than the ontological understanding of what is already present in QM, a new concept can be developed: "We claim that just like the logical and ontological principles of existence, non-contradiction and identity provide the constraints for a proper understanding of the concept of entity; the principles of indetermination, superposition and difference are able to determine the notion of power" (ibid). The concept of immanent ${ }^{2}$ power, then, shall be the central core of this new ontology, just as Aristotle's concept of entity was for his theory. Within the characterization of this concept we shall find the essence of this new ontology.

The first element of a Derondian power is its indetermination. This principle obviously stems from Heisenberg's relations, central core of QM, and seeks to understand that which is referred to by this postulate in its being indeterminate, rather than making of that indetermination an uncertainty, i.e., an epistemological/subjective problem. Indetermination is then an ontological characterization of a specific type of being. Powers are not entities: this means that they are not actual, they are not determined and they are not the substratum of classical properties. "The mode of being of a power is potentiality" [7, p. 12], it is probably in this statement where the true ontological innovation lies. A power is a potential being, as opposed to actual beings, and yet this potentiality is not to be understood in reference to actuality. In Aristotelian metaphysics potentiality, though a mode of being in its own right, cannot be without the actual. This is ultimately expressed in Aristotle's postulation of the first unmoved motor, which in its being perfect is pure act, no potentiality. So, a power must be understood as a being in its own right, which is in the mode of the potential and which is, in that potentiality, ontologically independent (from actuality). This means that both the realm of the potential, as its inhabitants, the powers, are ontologically separate and independent from the actual realm and actual entities. In the same way that entities inhabit actual space-time and in their being related to other entities and properties are part of what is called 'state of affairs', powers in their being related to other powers (and entities as well, since ontological independence does not mean absence of relations) belong to what de Ronde calls "potential states of affairs". ${ }^{3}$

The second principle that determines a power is that of superposition, another known tenant of QM. Superposed states are a normal currency in the quantum domain and yet, as famously shown by Schrödinger and his cat, the notion of having two mutually contradictory states superposed with one another is, from the perspective of a classical ontology, simply senseless. Superpositions seem to violate the principle of non-contradiction and I say 'seem', because the only way to violate the

\footnotetext{
${ }^{2}$ The "immanence" is related to the understanding of the measurement process in terms of the immanent cause. This is explained in detail in: [10].

${ }^{3}$ In order to make the distinction more explicit de Ronde refers to what was usually known as simply 'state of affairs' as 'actual state of affairs'.
} 
principle is if we accept it as operating. De Ronde's claim is that this principle is not valid in the quantum domain, since powers as being existents in the mode of the potential do not 'collapse' when 'supporting' mutually contradictory properties. The analog for a classical binary truth valuation to the elements $\{0,1\}$ is in this new ontology a potentia, that is, a measure for the intensity of a power: powers have definite potentia and they can be, from the perspective of actuality, contradictory, but from the perspective of the quantum we say they are simply superposed.

The third and last fundamental principle is that of difference as opposed to the Aristotelian identity. A power cannot be ascribed identity through time, inasmuch as it is not a temporal being and hence its unity, both conceptual and ontological, cannot rely in a spatio-temporal identification nor in a property-bundle theory. In this sense, we should think of a power as a being that does possess a unity that can be scientifically described, but not a strict identity as an actual entity would. The unity of the power is then found in difference, in that they change from a temporal perspective or are superposed. It should be noted, however, that the issue of identity is an extended metaphysical problem that applies as well to actual entities. Spatio-temporal beings also change through time, and the notion of identity through time requires some kind of metaphysical commitment: essence, material constitution, etc.

From this it follows, as anticipated, that powers, being in the mode of the potential, do not belong to the realm of the spatio-temporal: "A power cannot be thought as existing in space-time. It is only in the process, through which the power is exposed, that space-time enters the scene. The process builds a bridge to bring the power from its potential existence into its space-time actual effectuation" [7, p. 1]. Powers are not space-time beings, but we are, and so are the experiments we perform. For this reason, even if we speak about beings outside space-time, we do it from inside. We might occasionally evade space (in abstract mathematical reasoning, for example), but time is for us constitutive of our conscious existence. In this sense, we should not take our human limitation as an objective property of all being. Powers are non spatio-temporal, even if our means of experimentally accessing them are, and even if in our getting to know these powers empirically we 'change' them and submit them to our type of actual being. When we do so, we must understand that we access only a very partial aspect of these beings that are of a very different nature. 'Just as' we can only see an entity from one perspective at a time, never being able ever to stand from God's vantage point, our access to powers is an access to just one perspective of them, in this case though, probably much more limited than when we only see a face of a body.

As a way of summing up, I quote de Ronde's own self-valuation:

We would like to remark the fact that our notion of immanent power is maybe the first physical notion to be characterized ontologically in terms of an objective probability measure. This concept escapes the ruling of actuality since it is founded on a different set of metaphysical principles to that of classical entities. Indeed, powers are indetermined, paraconsistent and contextual existents. Powers can be superposed and entangled with different - even contradictory - powers. A power, contrary to a property which can be only true or false possesses an intrinsic probabilistic measure, namely, its potentia. A potentia is intrinsically statistical, but this statistical aspect has nothing to do with ignorance. It is instead an objective feature of quantum physical reality itself. [8, p. 34]

Now, there are several evidently controversial aspects about this proposal and hence the present work, in the hope of elucidating them. Here, I shall only enumerate these aspects, the analysis of which will be left for the final section:

I. Non-spatio-temporal physical beings: De Ronde's power is obviously not the first being outside of space-time that has been proposed. Plato's ideas could be considered as such. In 
the context of QM, also Aerts has recently proposed to consider non-spatial existents [1]. Even if it turns into a matter of beliefs and acceptance, we cannot simply discredit this possibility by assuming a materialist ontology. There is nothing particularly controversial about beings outside space-time, even if one would rather not accept their existence. What is highly controversial is considering beings outside space-time as physical existents that can be studied and explained by an empirical science such as physics, which is exactly what Derondian powers strive to be: the ontological concept of what occurs in the quantum domain, i.e., a domain of natural being studied by the physical science, formulated as QM.

II. Contradictory beings: even if we speak of powers instead of entities and of potentia instead of property, the possibility of something we understand as a comprehensible unitary being, being contradictory, requires more explanation. De Ronde's thesis is undoubtedly controversial and we require more than new names in order to accept the idea that we can think, explain and describe beings that are in their nature indeterminate and contradictory. ${ }^{4}$

III. Unity without identity: if we are to accept the claim that powers are indeed existent beings to be known and yet they do not possess self-identity, we need a clear substitute, for the concept to be meaningful. If the claim is that these beings possess some sort of unity that allows us to speak about powers as beings, and yet there is no possible identity to be ascribed to them, we need something that will stand in its place and offer an ontological unity for these powers. Even the concept of event which is clearly not an entity, has clear conditions of identity. This point violates Quine's famous dogma of "no entity without identity", and if we simply answer that we are not discussing entities then we are back to point $\mathbf{I}$. of this list.

A first reaction to this controversial theory is to deny it as implausible. Doing so is not hard, there are plenty of philosophers that could help in that task. The one that would do it better is probably Kant, as we can appreciate in Pringe's very thorough Kantian reading of Bohr's interpretation of QM:

If the quantum postulate is assumed, all pretension of reaching a spatial-temporal representation, which is at the same time causal, of an object subject to the postulate, must be abandoned. That is, if an object is within the domain of validity of the postulate, it won't be possible - as it is in classical physics - to synthesize the set of contingent data of a measurement, according to the concept of cause, as the effect of said object, representing this in space and time, in such a way that its states modify each other causally. [25, p. 183]

As it was mentioned in the previous section, Kant's result in the Critique of pure Reason is that an object of possible experience is that which is given in impressions under the pure forms of space and time, and under the a priori concepts, called categories, that synthesize those impressions into coherent objects. Anything outside these conditions cannot be legitimately called an object and therefore, the idea of it being studied by an empirical science is completely out of the question. Kant's result applied to QM, as Pringe's reading of Bohr proposes, is that we need to understand the quantum domain under symbolic analogies and regulative principles grounded in our experience of the actual realm, that is the only realm we can really experience, the scientific correlate of which is classical mechanics. Hence, this alternative ontology, as any other which goes against Kant's conclusions, is mere nonsense. My only answer to this position is a very simple question: why settle with Kant?

\footnotetext{
${ }^{4} \mathrm{Da}$ Costa and de Ronde have proposed to consider quantum superpositions in terms of a paraconsitent logic in [4]. This has lead to an interesting debate with Krause and Arenhart. See e.g.: [2, 11].
} 


\section{$3 \quad$ Brief introduction to phenomenology}

Indeed, why should we settle with an 18th century philosophy, when other theories are available to us, which could help us to better understand the peculiar situation brought about by QM. In this section, I face a difficult challenge, that of schematically presenting some of the basic tenants of one of the most important theories of the 20th century - one of the richest in production and bibliography - to readers most likely not familiar with any of it. I shall try to steer clear of technicalities, discussions and disagreements, in the hope of being able to present some basic notions of the theory, that I can later apply to de Ronde's alternative ontology. I shall do this, then, in a way that would most probably upset most phenomenologists, but that I hope could serve as an introduction to those unfamiliar with the theory, who could, if so they wished, continue reading on their own.

Phenomenology was founded by Edmund Husserl in the turn of the 19th century. It has many stages, already within Husserl's work, and many more if we consider his disciples, direct and indirect, and all the later developments through the 20th century up until today. Husserl was a mathematician, whose original concern was that of explaining how it is that we come to have numerical concepts and mathematical thinking in general. After his first works on the mathematical-psychological issues, the Logical Investigations, the most important work of the so called 'early phenomenology', are mainly concerned with discrediting psychologism, the position that sustains that the laws of logic and mathematics are dependent on the psychological laws of human thinking. Around 1907 Husserl is said to have taken a 'transcendental turn' in his philosophy, moving away from his early realism-empiricism to a transcendental philosophy. Ideas pertaining to a pure Phenomenology and to a phenomenological Philosophy [21] or simply Ideas I as it is usually referred to, is the work in which we find Husserl's transcendental static phenomenology explained. This we could call, not without some controversy, 'classic' or 'standard' phenomenology. It is mainly in this theory, with the backing of some later texts - mainly the Cartesian Meditations [20] - that I will be basing myself for this succinct presentation.

To begin with, let us characterize phenomenology in the most general terms. We can say phenomenology is a method that consists of describing experience such as it is experienced by the living subject. The first step in this method is to abandon the 'natural attitude' in which we live, and enter a critical attitude that will allow us to perform these descriptions from an objective standpoint. The phenomenological method consists in performing what Husserl called 'epoché', taken from the Greek word that means 'suspension of judgement'. Phenomenological epoché consists of suspending the 'effectivity thesis of the existence of the world', which means that, when we enter a reflexive attitude in order to describe our experience, we must ignore the actual existence of the world and its objects. In doing so, we access our own experience as such, without concerning ourselves with actuality, truth or correspondence. The basic, most important property of consciousness we immediately discover is that consciousness is always consciousness of something. This property is called intentionality. Consciousness is directed towards an object that is given to it as a correlate of experience. ${ }^{5}$ The results of this method claim a priori transcendental validity: "The epoché can also be said to be the radical and universal method by which I apprehend myself purely: as Ego, and with my own pure conscious life, in and by which the entire Objective world exists for me and is precisely as it is for me" [19, p. 21]. Transcendental phenomenology claims that the world and its objects (or phenomena) are constituted by the subject, but this constitution is not, so to say, arbitrary or relative, but objectively guided by the worldly objects themselves. The meaning of this is, that we cannot experience the world by any other way than that in which we constitute it, ${ }^{6}$ but

\footnotetext{
5 "In general, it belongs to the essence of each actual cogito to be consciousness of something" [21, p. 73].

6 "All real unities are unities of sense" [21, p. 120].
} 
the fact that we do constitute does not mean that there is no world, or that the world is relative to each constituting individual, since, precisely, in the phenomenological epoché we are describing transcendental properties of consciousness and not psychological properties of empirical individuals.

I would like to present next three phenomenological themes which are of great importance for the theory, and which I will be applying to de Ronde's theory of powers.

The first of these is the so called noetic-noematic structure of consciousness, also the a priori correlation. What this means is not so obscure as it might seem: by 'noesis' we understand what pertains to the mental acts (intentional acts) that consciousness performs. By 'noema' we understand the objective correlate of said acts, such as they are constituted by the intentional acts. The thesis of the correlations runs as follows: "Thus the eidetic law, confirmed in every case, states that there can be no noetic moment without a noematic moment specifically belonging to it" [22, p. 226]. The basic idea, as anticipated, is that constitution involves attributing sense to the objects we experience, and this phenomenon, rather than make our experience relative, it makes it possible:

Like perception, every intentive mental process — just this makes up the fundamental part of intentionality - has its 'intentional Object,' i.e., its objective sense. Or, in other words: to have sense or "to intend to" something is the fundamental characteristic of all consciousness which, therefore, is not just any mental living whatever, but is rather a $<$ mental living $>$ having sense, which is 'noetic'. [22, p. 217]

Noetically speaking, an act has different possible modes: one can perceive an object, remember it, desire it, fantasize it, imagine it, etc. For any act that consciousness performs, there must be a noematic correlate. The noema is an object such as it is constituted by the I, with the meaning invested upon the sensible impressions that we passively receive. We can never perceive all faces of a given object at the same time, nor can we perceive an object forever, without interruption. The perceived sides are said to be given, and the non-given sides are "apperceived" and intuited through acts of presentification. Both the given and not given "adumbrations" (Abschattungen) of the object are synthesized into unity. Perception is the paradigmatic act of consciousness, which gives us the object 'in the flesh', such as it is. Upon perception are founded all other acts, ideally speaking, which Husserl calls 'presentifications'. These are intuitive acts that give us the object not in the flesh, but in some other form (memory, imagination, etc.).

The second element I wish to present is merely an aspect of the general thesis of the correlation and it is that of the correlation between belief-characteristics and being-characteristics. In any intuitive act, noetically speaking, our act has a belief-characteristic that depends on the conditions under which we are intuiting, how well in particular we can intuit the given object, on attention and other noetic aspects. Noematically, to each corresponds a being-characteristic, as that with which the object is given to us. We can see this correlation in figure 1 :

\begin{tabular}{|c|c|}
\hline Belief (noetic) & Being (noematic) \\
\hline Certainty & $\begin{array}{c}\text { Truth - the object 'pure } \\
\text { and simple' }\end{array}$ \\
\hline Suspicion & Probability \\
\hline Conjecture & Possibility \\
\hline Question & Questionability \\
\hline Doubt & Dubious \\
\hline
\end{tabular}

Figure 1:

Certainty and truth are, respectively, the mother-forms of all characteristics, which also serve as 
ideals in guiding the different acts. If we take as example any simple case of perception, we can see how this works: in the middle of the night, on a dim-lighted street, we see what appears to be 'something' moving in the distance. We doubt whether or not there's something really there, and the 'something there' is for us of a dubious nature. Some moments later, a figure forms and we can no longer doubt whether there is or isn't something really there. We conjecture what it is. It is the possibility of a person approaching, as it would seem due to the way it moves. As it gets even closer and passes under a lamppost we reach the strong suspicion that this is most probably a human being, who finally stands before us, asks us for a cigarette, and we reach the certainty that, truly, there is a human being in the flesh standing right there. ${ }^{7}$

The last point I wish to present is that of empathy. Empathy is a specific form of constitution that differs from the constitution of other objects of our experience. Empathy is the intentional act through which we constitute 'others as myself', other human beings, conscious subjects. The main difference is that, in this case, we cannot access that which defines the thing as what the thing is: we cannot perceive the stream of experiences of others, it is never given to us in the authentic intuition of perception. Therefore, we must do something else in order to know that these special objects that stand before us in our life are not mere bodies, like other physical objects, but are in fact consciousnesses that have experience of the world just as we do. In order to reach this knowledge, we ground our acts in the perception of the body of the other. The body is given to us, just as any other body is. When we perceive any physical body, say a tree, we see one side of it. The other sides, not actually being perceived, are said to be appresented, which means that they are not being perceived at the moment, and could be either presentified (i.e. imagined) or empty, but could be eventually perceived, if one just moves around the tree and sees it from the opposite side. In the case of empathy, though, the stream of consciousness of the other is appresented, but can never be perceived. That appresentation can only be fulfilled in a presentification. So, in order to reach this level (what Husserl calls authentic empathy) we need something other than the mere perception of a body. We see, for example, how that peculiar body is not just there, like it happens with tables and stones, but it also moves. Not only it moves, but it seems to move in a very specific way, i.e. like I move, with purpose. We see that body interacting within nature, with other bodies in a way we can consider analogic to our own way of moving and interacting (what Husserl calls 'parification'). We also see that this body speaks, and in doing so, expresses beliefs, desires, goals, fears, etc. We reach authentic empathy when we can presentify in imagination all that conscious life we cannot perceive, and understand that there is someone who has experience of the world, in the same sense as I do. In superior forms of empathy, which require the intervention of active reasoning, we can presentify the specific mental experiences of a concrete individual, what this particular person wants, believes, loves, etc. ${ }^{8}$

With this, we move on to the analysis of de Ronde's theory, under the phenomenological perspective.

\section{Phenomenology and immanent powers}

I would like to begin with a general consideration that stems from the phenomenological thesis of the noetic-noematic correlation. De Ronde offers in his theory the rudimentary principles to build a new ontology that shall serve as the basis for QM. In this line, we could say that he is describing a

\footnotetext{
${ }^{7}$ Cf. [22, pp. 249-252].

${ }^{8}$ For Husserl's most systematic presentation of Empathy, see the $V$ Cartesian Meditation [19], [20]. An excellent work (in Spanish) on the subject is [26].
} 
specific ontological region, ${ }^{9}$ inhabited by certain type of noemata: immanent powers with a definite potentia. We find in his theory (leaving aside how developed or in need of development it might stand) the formal principles to describe such an ontological realm. We could say, then, that they stand on the noematic side of the correlation. What we are still missing is a noetic description of what acts would be involved in the constitution of said noemata. Which acts must consciousness perform in order to constitute meaningful noemata, such as the Derondian powers?

A first answer to this problem, in strong contraposition to a Kantian approach, is that it is possible to find this noetic description. For Kant, objective experience is limited by impressions and concepts. In the absence of one we have no object, and hence no possibility of science. Phenomenology's concept of what can be objective experience is much, much broader than that. It includes the possibility of everything that consciousness experiences, lives, of what is given to it. Perceptual-physical objects are one domain of experience. Mathematical, abstract thinking is another, art and society yet others. As long as we can find the noetic acts involved in the specific type of constitution, we can legitimately speak about experience. If we can't, it would mean that the discourse about powers is indeed 'empty talk', just words with no reference, with no kind of intuitive comprehension whatsoever. So, the first point to claim from the phenomenological perspective would be: there is no a priori impediment against the notion of a Derondian power — as there would be from a Kantian perspective. Of course, it is yet to be seen whether it is in fact phenomenologically plausible to constitute the proposed beings.

Now the second question to answer phenomenologically is: is it possible to constitute these contextual, superposed, different, outside space-time, even contradictory beings, which we cannot even perceive? Again, a first phenomenological answer would be: it is possible. Here is where empathy comes in. I have brought this theme from phenomenology precisely for this particular point. In the case of empathy, we constitute very specific beings, under quite peculiar conditions. As it was explained, the consciousness of the other is never given to perception. Yet, at least from the phenomenological perspective, its reality cannot be denied. We know these funny bodies that walk about are conscious entities such as myself, even if I can never access their consciousness directly, and even if I sometimes wonder whether I am alone in the universe.

What I want to state is that empathy is not only an example of successful constitution without direct perception, but it is also an example of constitutions that involve contradictory (superposed) beings. When we come to the higher forms of empathy and reflect upon the specific conscious life of a particular individual, we are faced with something quite similar to a superposition state. What does this person want? Does she love me? Will he accept my offer? We could consider this mere ignorance regarding our knowledge of the other person's state of mind, and in many situations, this might very well be the case. But if we reflect upon our own beliefs, desires, projects, ambitions, we can very easily verify that in many other cases the problem is not one of ignorance, but of actual indetermination. We do not know what we want. We want something, but don't want the means it implies, or some yes, but not all, and we are afraid of the consequences, but we would really like it to turn out this way? So? What is the answer, in a specific situation, to the question "does $\mathrm{X}$ want Y?". Funny as it might sound, one way of expressing it could be: $\mathrm{X}=60 \%$; Y $=40 \%$. And then we ask, and find in actuality only one answer, because that is all that actuality tolerates, one

\footnotetext{
${ }^{9}$ The concept of ontological region is presented by Husserl in Chapter 1 of Ideas I. It is a complicated concept, as the whole chapter is. The concept is presented as being defined by a kind of object, whose ontological properties are unique. The mother-forms of ontological regions are Nature and Consciousness. Nature is defined by being spatiotemporal and Consciousness by being only temporal. Thus, another form to present de Ronde's claim is precisely the idea that immanent powers constitute a unique type of object (the concept of object is not to be confused with that of entity, object in phenomenology is the most general category to speak about "something") and therefore belong to a distinct ontological region, different from that of the macroscopic objects described by classical physics and experienced in the natural attitude.
} 
definite state. But our consciousness, though invariably and inexorably bound by temporality, does not inhabit physical space and is not susceptible to the deterministic laws of classical mechanics. ${ }^{10}$ We can be, as we so often are, in a state of indetermination, superposed by contradictory desires. And being this the way it is, we can still successfully constitute alter-egos as coherent, unitary beings and say: this is John, he has experience of the world just like I do, even if I don't see his experience. He is a catholic, but he also believes that abortion is right (contradiction), he says he wants to come to the party on Saturday, but he also says he might not come; I know he is my friend, even though sometimes he is bad to me, etc., etc. John is a coherent unitary being, made of contradictions and superpositions which I can only presentify and never perceive.

It is not my claim, under no circumstance and in no sense, that human consciousness are quantum superposition states. My only claim is that we cannot rule out the possibility of constituting something like a Derondian power, 'just because' it is outside normal macroscopic perception, because it involves the superposition of mutually contradictory states. We do this already in the case of the empathic constitution of the alter-ego, and we do it successfully. It is my claim, then, that if it is possible in one domain, it might be possible in another, such as the quantum realm.

Two problems in this direction still stand. In the case of empathy, as we saw, the constitution of the other is founded upon the perception of the physical body. There is a perception that serves as basis for the presentification of the not-given aspects, in this case, the conscious life itself. What is the founding perception in the case of powers? This question remains unanswered. We need something that serves as basis and guide for the constitution of other elements. We cannot simply do everything in presentification, because that would be mere imagination and we can hardly call that science. A possible candidate could be the actual, already performed, experimental arrangements. The problem is that they are incompatible with de Ronde's pretentions. According to him, in experimental arrangements we only access the actualized power, an actual effectuation, but not the power itself, nor the power in its most important form, which is that which is not actualized. We need a good candidate to serve as a basis and guide for a successful constitution of a power, even if many or most of its sides remain only appresented and fulfilled in presentification, as is the case of empathy.

The second associated problem, much harder to solve, is that which I listed as number 1: powers are not actual, but they are physical. In phenomenological terms, real means space-time bound, while ideal means outside space-time. Ideal entities are obviously much more tricky than real ones, but they are nothing to discourage us: mathematical entities, meanings, essences, species, are all more or less familiar ideal entities. This poses a huge ontological problem: we are intersecting what up to now were two completely separated realms of being, the 'ideal' (in the Husserlian sense) and the physical. The solution to this problem is, I believe, intimately bound to the previous point: if something, say a Derondian power, belongs to physical nature, no matter what other peculiarities it has (being outside space-time, being contradictory, etc.) there must be some sort of perception of it, or of one of it sides. Otherwise, all concepts lose meaning. I represent this problem in the following scheme:

The remaining point concerns the phenomenological notion of belief- and being-characteristics, and how this can be applied to the issue of identity and truth in regards to powers. As we saw, de Ronde claims that the information we obtain when we perform a measurement is only very

\footnotetext{
${ }^{10}$ It is worth noting that the attempts to clarify the phenomenon of empathy (or theory of mind) in other philosophical theories and other disciplines, such as cognitive sciences, would state something quite different, if not the exact opposite. It is therefore also worthy of attention, that these programs that accept naturalistic principles for the study of the human "mind" incur in the same attitude as the philosophers of physics that pretend to limit QM to the classical ontology: they accept only one realm of the real (actual, material existence) and only one method to study it (logical-empiricism, observability).
} 


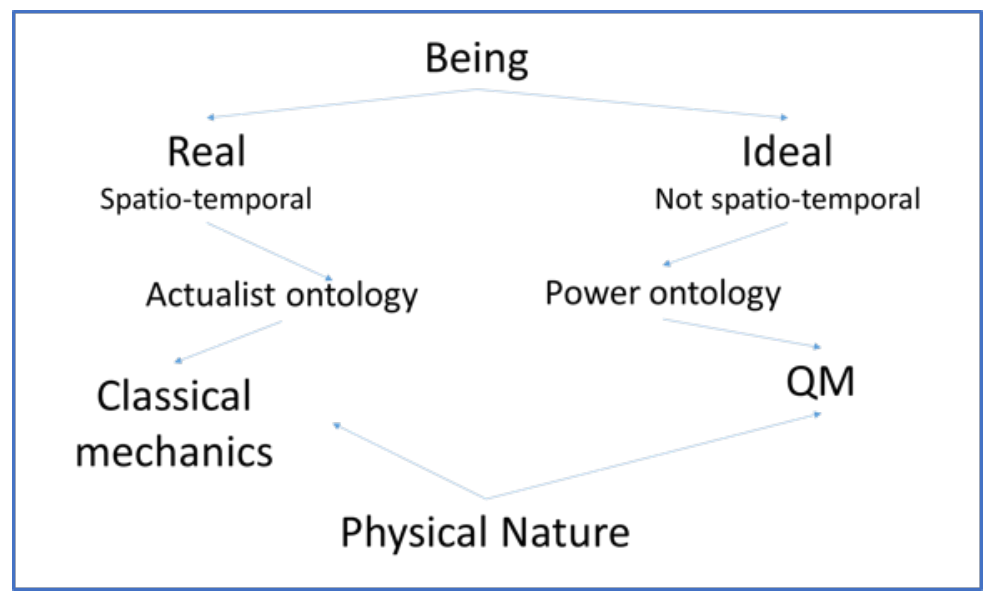

Figure 2:

partial regarding the ontological existent beneath it, which is the power. Yet, due to contextuality, our knowledge of the power itself can never be expressed in terms of a 'truth or false' ' 1 or 0 ' valuation: "Quantum contextuality, which was most explicitly recognized through the KochenSpecker theorem, asserts that a value ascribed to a physical quantity A cannot be part of a global assignment of values but must, instead, depend on some specific context from which $\mathrm{A}$ is to be considered" [12, p. 5],. In this sense, as the problem was listed under number 3 , we need new criteria to determine the identity of a power and a new corresponding notion of truth to value our statements about powers:

Our proposed representation of quantum physical reality in terms of powers with definite potentia opens the possibility of considering a reference which is not exclusively defined in terms of 0 or 1 values (true or false). Instead, the power can be understood as possessing an intensity, or in other words, a potentia which pertains to the closed interval $[0 ; 1]$. According to our approach, given a $\Psi$, there is a set of powers which can be defined as being true in the ontic level but, contrary to the classical case, their relation to actual effectuations is not that of a one-to-one correspondence. Indeed, due to the fact that a power has assigned a potentia in the closed interval [0,1], statistics is required in order to acquire complete knowledge of each power. In order to gain knowledge about a PSA we require the (contextual) measurement of each (non-contextual) power and its potentia. Thus, while classical physics has a binary valuation of properties, QM presents instead an intensive valuation of powers. [12, p. 18]

Is this possible? Can we legitimately keep talking about truth in the absence of absolute values? Let's analyze the problem from the phenomenological perspective, taking into consideration the correlation between belief and being characteristics. The essential law we quoted above states there must always be a correlation between the noetic (belief) and the noematic (being), in other words, to every noetic element, there must always correspond a noematic one. The actual correlation between characteristics we saw above is an example of this more general noetic-noematic correlation, and it is also an application to a known domain, paradigmatically that of perception. Now, as long as we do not violate the essential law and maintain the correlation, we could revise how this specific correlation is, as a matter of fact, for the quantum domain. I mean the following: due to the potential ontological nature of powers and potentia, known to us by the Born rule and contextuality, and QM in general, we could then claim that in QM, what corresponds to certainty is not actual truth, 
but a probability value. And what corresponds to truth, in the sense of something fully given in actuality, is not certainty, but a conjecture regarding the full nature of the power that can only be partially known in an actual measurement; changing some of the correspondences in the table, so as to have:

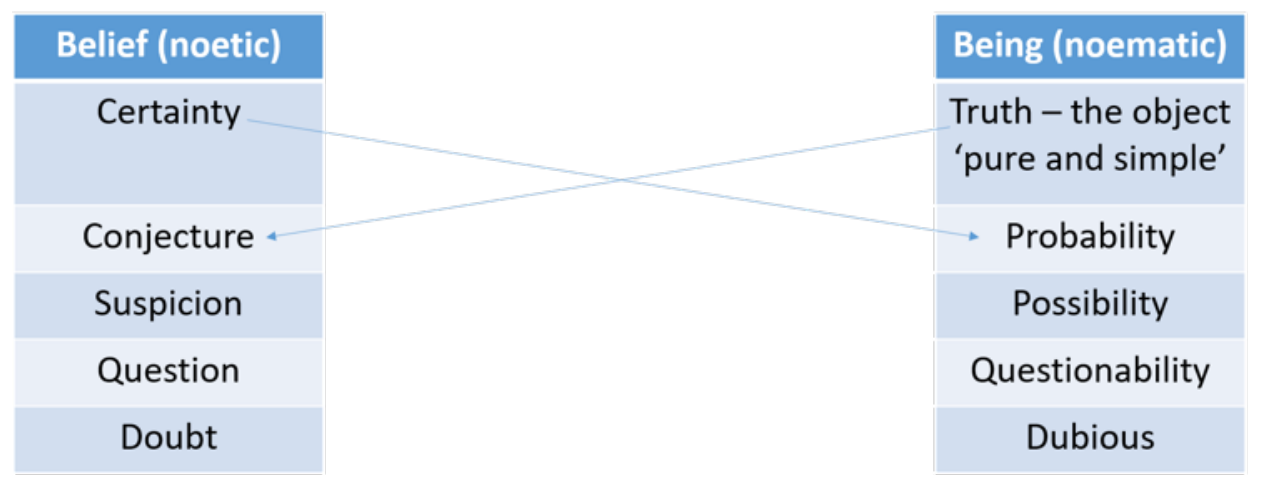

Figure 3:

The idea would then be that we have certainty, certain knowledge of a quantum power, when we know it in its being probabilistic and indeterminate and not when it is given in a determined value in the actuality of a measurement process. Through an experimental result, we access only a partial aspect of the power, and therefore can only suspect what the true nature of the power really is. A power can then be identified by its intensive probability value, which is a specific value that is assigned to the power, even though it is not a determinate value.

Of course, this is not enough to fully determine the identity of a power, and neither is the correlation a definitive notion of truth. But again, we can appreciate the plausibility of utilizing new notions of truth and identity to describe a domain that is, in itself, radically new. Regarding the violation of Quine's dogma, all we can say is: of course! We are not talking about entities, but about powers!

\section{Conclusions}

In this article, I have surveyed the notion of an alternative ontology to QM, a necessity that stems from the impossibility of describing what QM talks about. Most attempts to do so, have done it in the line of maintaining themselves within the limits of classical ontology and classical mechanics, even at the expense of the formalism itself. When it comes to a new ontology, alternative to the classical one, we are immediately faced with a myriad of problems. I have presented de Ronde's alternative ontology of powers and potentia, and I have analyzed it from the phenomenological perspective. My conclusion is, then, quite simple: I do not claim that de Ronde's ontology is the correct one for QM (that question falls out of the scope of my work). Neither do I claim that de Ronde's ontology is finished or without problems to be revised. What I do claim is that there is no a priori restriction for the possibility of said ontology, that it does not violate any phenomenological restriction. This not only clears the way to keep working on this project, but it also helps to point out what the problems to be solved are. In particular, I would like to highlight two: the need to find an element in the order of the intuitive (noetic) that would allow and guide the proper meaningful constitution of the power itself; and the huge problem that implies considering an ontological realm that is not spatio-temporal, and yet belongs to physical nature. I believe that, in what concerns the philosophical development of this ontology, these are the major challenges for the theory. 


\section{Acknowledgements}

This work was completed under a DAAD grant. I would like to thank Christian de Ronde for the discussion and valuable comments on the manuscript.

\section{References}

[1] Aerts D., 2010, "A potentiality and Conceptuality Interpretation of Quantum Physics", Philosophica, 83, 15-52.

[2] Arenhart, J. R. and Krause, D., 2016, "Contradiction, Quantum Mechanics, and the Square of Opposition", Logique et Analyse, 59, 273-281.

[3] Bitbol, M., 2010, "Reflective Metaphysics: Understanding Quantum Mechanics from a Kantian Standpoint", Philosophica, 83, 53-83.

[4] da Costa, N. and de Ronde, C., 2013, "The Paraconsistent Logic of Quantum Superpositions", Foundations of Physics, 43, 845-858.

[5] D'Espagnat, B., 1989, Reality and the Physicist: Knowledge, duration and the quantum world, Cambridge University Press.

[6] de Ronde, C., 2011, The Contextual and Modal Character of Quantum Mechanics: A Formal and Philosophical Analysis in the Foundations of Physics, $\mathrm{PhD}$ dissertation, Utrecht University, The Netherlands.

[7] de Ronde, C., 2013, "Representing Quantum Superpositions: Powers, Potentia and Potential Effectuations", preprint. (quant-ph:1312.7322)

[8] de Ronde, C., 2016, "Probabilistic Knowledge as Objective Knowledge in Quantum Mechanics: Potential Powers Instead of Actual Properties", in Probing the Meaning and Structure of Quantum Mechanics: Superpositions, Semantics, Dynamics and Identity, pp. 141-178, D. Aerts, C. de Ronde, H. Freytes and R. Giuntini (Eds.), World Scientific, Singapore.

[9] de Ronde, C., 2017, "Quantum Superpositions and the Representation of Physical Reality Beyond Measurement Outcomes and Mathematical Structures", Foundations of Science, https://doi.org/10.1007/s10699-017-9541-z. (quant-ph:1603.06112)

[10] de Ronde, C., 2017, "Causality and the Modeling of the Measurement Process in Quantum Theory", Disputatio, forthcoming. (quant-ph:1310.4534)

[11] de Ronde, C., 2018, "A Defense of the Paraconsistent Approach to Quantum Superpositions (Reply to Arenhart and Krause)", Metatheoria, forthcoming. (quant-ph:1404.5186)

[12] de Ronde, C., 2018, "Potential Truth in Quantum Mechanics", preprint.

[13] Dorato, M., 2015, "Events and the Ontology of Quantum Mechanics", Topoi, 34, 369-378.

[14] Esfeld, M., 2009, "The modal nature of structures in ontic structural realism", International Studies in the Philosophy of Science, 23, 179-194. 
[15] French, S., 2015, "Doing Away with Dispositions: Powers in the Context of Modern Physics", in Dispositionalism: Perspectives from Metaphysics and the Philosophy of Science A. S. MeinckeSpann (Eds.), Springer Synthese Library, Springer.

[16] Fuchs, C. and Peres, A., 2000, "Quantum theory needs no 'interpretation"', Physics Today, 53, 70 .

[17] Griffiths, R., 2013, "A Consistent Quantum Ontology", Studies in History and Philosophy of Science Part B, 44, 93-114.

[18] Graffigna, M., 2016, "The Possibility of a New Metaphysics for Quantum Mechanics from Meinong's Theory of Objects", in Probing the Meaning and Structure of Quantum Mechanics: Superpositions, Semantics, Dynamics and Identity, pp. 280-307, D. Aerts, C. de Ronde, H. Freytes and R. Giuntini (Eds.), World Scientific, Singapore.

[19] Husserl, E., 1960, Cartesian Meditations: An Introduction to Phenomenology. Martinus Nijhoff, The Hague.

[20] Husserl, E., 1973, HUA I: Cartesianische Meditationen und Pariser Vorträge. S. Strasser (Eds.), Martinus Nijhoff, The Hague.

[21] Husserl, E., 1977, HUA III-I: Ideen zu einer reinen Phänomenologie und phänomenologischen Philosophie. Erstes Buch: Allgemeine Einführung in die reine Phänomenologie 1. Halbband: Text der 1.-3. Auflage - Nachdruck, Karl Schuhmann (Eds.), Martinus Nijhoff, The Hague.

[22] Husserl, E., 1983, Ideas 1, Martinus Nijhoff, The Hague. (Originally published in 1913)

[23] Kant, I., 1998, Kritik der reinen Vernunft, Akademie Verlag, Berlin.

[24] Lurat, F., 2007, "Understanding Quantum Mechanics", in Rediscovering Phenomenology, L. Boi, P. Kerszberg, P. and F. Patras (Eds), Springer, Dodrecht.

[25] Pringe, H., 2012, "La filosofía trascendental y la interpretación de Bohr de la teorá cuántica", Scientiae Studia, 10, 179-194.

[26] Walton, R., 2001, "Fenomenologa de la empatía", Philosophica, 24, 25.

[27] Wheeler, J. A. and Zurek, W. H. (Eds.) 1983, Theory and Measurement, Princeton University Press, Princeton. 